\title{
Giant Magnetic Anisotropy of Single Cobalt Atoms and Nanoparticles
}

\author{
P. Gambardella, ${ }^{1,2 *}$ S. Rusponi, ${ }^{1,2}$ M. Veronese, ${ }^{3}$ S. S. Dhesi, ${ }^{4} \dagger$ \\ C. Grazioli, ${ }^{3}$ A. Dallmeyer, ${ }^{5}$ I. Cabria, ${ }^{5}$ R. Zeller, ${ }^{5}$ \\ P. H. Dederichs, ${ }^{5}$ K. Kern, ${ }^{1,2}$ C. Carbone, ${ }^{3,5}$ H. Brune ${ }^{1}$
}

\begin{abstract}
The isotropic magnetic moment of a free atom is shown to develop giant magnetic anisotropy energy due to symmetry reduction at an atomically ordered surface. Single cobalt atoms deposited onto platinum (111) are found to have a magnetic anisotropy energy of 9 millielectron volts per atom arising from the combination of unquenched orbital moments (1.1 Bohr magnetons) and strong spin-orbit coupling induced by the platinum substrate. By assembling cobalt nanoparticles containing up to 40 atoms, the magnetic anisotropy energy is further shown to be dependent on single-atom coordination changes. These results confirm theoretical predictions and are of fundamental value to understanding how magnetic anisotropy develops in finite-sized magnetic particles.
\end{abstract}

Permanent magnets play a key role in technology and industry, ranging from the generation and distribution of electrical power to communication devices and the processing of information $(1,2)$. The property that makes them so useful is the magnetic anisotropy energy (MAE), which describes the tendency of the magnetization to align along specific spatial directions rather than randomly fluctuate over time. The MAE determines the stability of the magnetization in bulk as well as nanoparticle systems. Extensive studies on ferromagnetic bulk materials and thin films have highlighted the MAE dependence on crystal symmetry and atomic composition (1). Whereas the exchange interaction among electron spins is purely isotropic, the orbital magnetization, via the spinorbit interaction, connects the spin magnetization to the atomic structure of a magnetic material, hence giving rise to magnetic anisotropy $(3,4)$. With respect to bulk solids, surfacesupported nanoparticles offer additional degrees of freedom to tune the MAE by ad hoc modifications of the particle size, shape, and coupling with the substrate, making nanosized systems attractive for basic investigations as well as for miniaturized data-storage applications $(5,6)$. However, fundamental points re-

${ }^{1}$ Institut de Physique des Nanostructures, Ecole Polytechnique Fédérale de Lausanne, $\mathrm{CH}-1015$ Lausanne, Switzerland. 'Max-Planck-Institut für Festkörperforschung, Heisenbergstrasse 1, D-70569 Stuttgart, Germany. ${ }^{3}$ Istituto di Struttura della Materia, Consiglio Nazionale delle Ricerche, Area Science Park, I-34012 Trieste, Italy. ${ }^{4}$ European Synchrotron Radiation Facility, BP 220, F-38043 Grenoble, France. ${ }^{5}$ Institut für Festkörperforschung, Forschungszentrum Jülich, D-52425 Jülich, Germany.

*To whom correspondence should be addressed. Email: pietro.gambardella@epfl.ch

$\uparrow$ Present address: Diamond Light Source, Rutherford Appleton Laboratory, Didcot OX11 0QX, UK. main unclear: how the MAE evolves from single atoms to finite-size magnetic particles, how it correlates to the atomic magnetic moments, and how both depend on the details of the atomic coordination.

Free transition metal (TM) atoms possess large spin (S) and orbital (L) magnetic moments according to Hund's rules. Conversely, the survival of localized magnetic moments on TM impurities dissolved in nonmagnetic metal hosts is a long-debated problem in solid-state physics $(7,8)$. Electron delocalization and crystal field effects compete with the intra-atomic Coulomb interactions, responsible for Hund's rules, causing a substantial or total decrease of $\mathbf{S}$ and quenching of $\mathbf{L}$. Theoretical calculations, however, predict such effects to be strongly reduced at surfaces owing to the decreased coordination of TM impurities, with implications also for the appearance of substantial magnetic anisotropy (9). The determination of $\mathbf{S}$ and particularly of $\mathbf{L}$ and the MAE for isolated surface adatoms, however, has been hampered by the lack of experimental techniques with the required sensitivity. Gas-phase experiments have shown a strong dependence of the per-atom magnetization on particle size in TM clusters containing less than 500 atoms (10). Changes in the magnetization are therefore expected also for particles of reduced size grown on a metal substrate. We report on a bottom-up approach to study how the magnetization and MAE develop in TM particles constructed on a nonmagnetic substrate. We start from isolated magnetic atoms deposited on an atomically ordered metal surface and subsequently increase the particle size almost in an atom-by-atom fashion. The observation of $\mathbf{L}$ and the MAE in single-surface adatoms and small particles in this critical size range are reported and compared with ab initio calculations.

The Co atoms and particles were deposited in ultrahigh vacuum by molecular beam epitaxy on a clean $\mathrm{Pt}(111)$ surface. The $\mathrm{Co} / \mathrm{Pt}$ system was chosen to maximize the MAE effects. Among 3d ferromagnetic elements, hexagonal close-packed (hcp)-Co presents the largest MAE, whereas CoPt alloys show a large MAE increase (11) owing to the strong spin-orbit coupling of the Pt $5 \mathrm{~d}$ states. Isolated adatoms were obtained by depositing minute amounts of Co [less than 0.030 monolayers (ML); $1 \mathrm{ML}=$ $1.5 \times 10^{15}$ atoms $\left./ \mathrm{cm}^{2}\right]$ at temperature $T=5.5 \mathrm{~K}$ to inhibit surface diffusion (Fig. 1A). Twodimensional, monolayer particles of larger size were obtained by statistical growth for average size $1<\bar{n} \leq 5$ atoms and by diffusioncontrolled aggregation $(12,13)$ at different $T$ values for $5<\bar{n} \leq 40$ atoms. The standard deviation of the size distribution determined by scanning tunneling microscopy (STM) was $\bar{n}^{1 / 2}$ and $0.3 \bar{n}$ in the first and second case, respectively (13). In the STM image of isolated Co adatoms on the $\mathrm{Pt}(111)$ substrate (Fig. 1A), the individual Co atoms are clearly visible as separate white dots. X-ray magnetic circular dichroism (XMCD) (14) measurements were performed to probe the adatom magnetism by detecting x-ray absorption spectra (XAS) at the Co $L_{2,3}$ edges ( $2 p$ to $3 \mathrm{~d}$ transitions) using left and right circularly polarized light in the total electron yield mode (Fig. 1B). The XMCD signal (Fig. 1C) is the difference between the XAS spectra recorded for parallel $\left(\mu_{+}\right)$and antiparallel $\left(\mu_{-}\right)$alignment of the photon helicity with the applied field $\mathbf{B}$. Fields of up to $7 \mathrm{~T}$ were used to magnetize the sample at angles $\theta_{0}=0^{\circ}, 70^{\circ}$ with respect to the surface normal. The adatom XAS spectra show relatively weak absorption features compared with the Pt background due to the extremely low concentration of Co adatoms. However, the XMCD signal is very large relative to the total Co XAS signal, indicating the presence of strong magnetic moments localized on the Co atoms. Two effects are representative of single-adatom magnetic properties. First, the vanishing intensity of the XMCD at the $L_{2}$ edge indicates unusually strong orbital magnetism; second, the large difference between the magnetization measured in-plane and outof-plane up to $7 \mathrm{~T}$ (Fig. 1D) reveals an extraordinary MAE.

According to the XMCD sum rules (1517), the projections of $\mathbf{L}$ and $\mathbf{S}$ along the incident light direction can be obtained from

$\mathbf{L}=-\frac{4}{3} h_{\mathrm{d}} \frac{\int_{L_{3}+L_{2}}\left(\mu_{+}-\mu_{-}\right) d E}{\int_{L_{3}+L_{2}}\left(\mu_{+}+\mu_{-}\right) d E}$ 


$$
\begin{aligned}
& \mathbf{S}+7 \mathbf{D}= \\
& -h_{\mathrm{d}} \frac{6 \int_{L_{3}}\left(\mu_{+}-\mu_{-}\right) d E-4 \int_{L_{3}+L_{2}}\left(\mu_{+}-\mu_{-}\right) d E}{\int_{L_{3}+L_{2}}\left(\mu_{+}+\mu_{-}\right) d E}
\end{aligned}
$$

where $h_{\mathrm{d}}$ is the number of holes in the Co $3 \mathrm{~d}$ states and $\mathbf{D}$ is the magnetic spin dipole moment. Along the easy direction $\left(\theta_{0}=0^{\circ}\right)$, we obtain $\mathbf{L}=1.1 \pm 0.1 \mu_{\mathrm{B}} /$ atom and $\mathbf{S}+$ $7 \mathbf{D}=1.8 \pm 0.1 \mu_{\mathrm{B}} /$ atom, using the value $h_{\mathrm{d}}=2.40$ calculated within the local spin density approximation (LSDA) for the specific case of Co adatoms on $\mathrm{Pt}(111)$. The adatom $\mathbf{L}$ is extremely high for a d-metal system, especially compared with bulk hcpCo $\left[\mathbf{L}=0.15 \mu_{\mathrm{B}}(17)\right]$, a two-dimensional Co monolayer on $\operatorname{Pt}(111)\left[\mathbf{L}=0.29 \mu_{\mathrm{B}}\right.$ (this work)], Au-capped Co clusters on $\mathrm{Au}(111)$ $\left[\mathbf{L}=0.30 \mu_{\mathrm{B}}(18)\right]$, and even one-dimensional Co monatomic chains $\left[\mathbf{L}=0.68 \mu_{\mathrm{B}}\right.$ (19)]. The explanation lies in the reduced coordination of an isolated atom adsorbed on top of a flat surface, which favors delectron localization and the survival of atomic-like character in the $3 \mathrm{~d}$ orbitals. Unfortunately, the value of $\mathbf{S}$ cannot be determined with equal accuracy by virtue of Eq. 2 because $\mathbf{D}$, which arises from the anisotropy of the atomic charge distribution, is unknown. However, contrary to the case for $\mathbf{L}, \mathbf{S}$ can be reliably calculated in the LSDA scheme, yielding $2.14 \mu_{\mathrm{B}}$, as detailed later. We therefore estimate $7 \mathbf{D} \approx$ $-0.34 \mu_{B}$, which is between the values expected for truly atomic-like d-orbitals and bulk $\mathrm{Co}(8,20)$.

We determined the MAE by measuring the adatom magnetization parallel to $\mathbf{B}$, with $\mathbf{B}$ applied along different directions with respect to the surface normal (Fig. 1D). In the paramagnetic case, the magnetization of an assembly of particles of size $\bar{n}$ can be calculated with the magnetic energy function $E\left(\theta_{0}, \theta, \phi\right)$, where $\theta$, $\phi$ are the polar and azimuthal coordinates of the total magnetic moment $\bar{n} \mathbf{m}, \mathbf{B}$ is taken as the $z$ axis, and $\theta_{0}$ defines the easy magnetization direction. $E$ is the sum of a Zeeman term $-\bar{n} \mathbf{m} \mathbf{B} \cos \theta$ and, assuming uniaxial anisotropy, of a MAE term of type $-\bar{n} K\left(\sin \theta_{0} \sin \theta \cos \phi+\right.$ $\left.\cos \theta_{0} \cos \theta\right)^{2}$, where $K$ is the MAE per Co atom. The magnetization then reads (21)

$$
\begin{aligned}
& M= \\
& M_{\text {sat }} \frac{\int_{0}^{2 \pi} d \phi \int_{0}^{\pi} d \theta \sin \theta \cos \theta e^{-E\left(\theta_{0}, \theta, \phi\right) k_{\mathrm{B}} T}}{\int_{0}^{2 \pi} d \phi \int_{0}^{\pi} d \theta \sin \theta \mathrm{e}^{-E\left(\theta_{0}, \theta, \phi\right) k_{\mathrm{B}} T}}
\end{aligned}
$$

where $M_{\text {sat }}$ is the saturation magnetization. The solid lines in Fig. 1D represent numerical fits of the data by means of Eq. 3. From the two curves one obtains the parameters $M_{\mathrm{sat}}, \bar{n} \mathbf{m}$, and $K$. The key result here is the magnitude of the MAE, which makes it very hard to align the adatom magnetic moment in the in-plane direction even at $\mathbf{B}=7 \mathrm{~T}$ (Fig. 1D). We find $K=9.3 \pm 1.6$ meV/atom, which is exceptionally large compared with any known magnetic material. Typical systems with high MAE are $\mathrm{SmCo}_{5}[K=$ $17 \times 10^{7} \mathrm{erg} / \mathrm{cm}^{3}=1.8 \mathrm{meV} / \mathrm{Co}$ atom (2)], $\mathrm{Co} / \mathrm{Pt}, \mathrm{Co} / \mathrm{Au}$ multilayers $[K \sim 0.3 \mathrm{meV} / \mathrm{Co}$ atom $(22,23)]$, and one-dimensional Co atomic chains $[K=2.0 \mathrm{meV} / \mathrm{Co}$ atom (19)]. This value of $K$ does not change appreciably with Co concentration for coverages between 0.007 and 0.03 ML ( $K=9.2$ and $8.6 \mathrm{meV} /$ atom, respectively). The total magnetic moment is given by $\mathbf{m}=$ $\mathbf{S}+\mathbf{L}+\mathbf{m}_{\mathrm{Pt}}$, where $\mathbf{m}_{\mathrm{Pt}}$ represents the magnetic moment induced on Pt sites per Co atom. For the isolated adatoms, we find $\mathbf{m}=5.0 \pm 0.6$ $\mu_{\mathrm{B}}$. By taking $\mathbf{L}=1.1$ and $\mathbf{S}=2.1 \mu_{\mathrm{B}}$, we obtain $\mathbf{m}_{\mathrm{Pt}}=1.8 \pm 0.7 \mu_{\mathrm{B}}$, where, as in the case of $K$, the error is mostly due to the uncertainty of the sample temperature. The large induced magnetization agrees with the fact that dilute Co impurities in bulk Pt are a well-known giantmoment system, where for a 0.5 to $1 \%$ atomic concentration one derives $\mathbf{m}_{\mathrm{Pt}} \approx 3.1 \mu_{\mathrm{B}}(24)$.
Different effects combine in establishing the large MAE of Co adatoms on the Pt surface. (i) The broken symmetry of the Co adatoms as compared with the free atom constitutes the necessary condition to exhibit anisotropic behavior. (ii) The reduced coordination leads to 3d-electron localization (band narrowing), which augments the spin-orbit energy due to increases in the local density of states near the Fermi level and the spin magnetic moment (3, 4). (iii) The strong spin-orbit coupling of the $\mathrm{Pt}$ $5 \mathrm{~d}$-states results in additional MAE of the induced magnetization, an effect common to $\mathrm{CoPt}$ compounds (25). Whereas (i) determines the angular dependence of the MAE, (ii) and (iii) determine its magnitude. The measurements on the larger Co particles further reveal the predominant influence of (ii) on the MAE and $\mathbf{L}$ magnitude.

Figure 2A shows how the XMCD spectra change for Co particles with average size between 3 and 40 atoms. The decrease of the XMCD intensity at the $L_{3}$ edge relative to $L_{2}$ is the signature of progressive quenching of $\mathbf{L}$ with increasing particle size. The MAE decreases in parallel, as can be seen by comparing the in-plane and out-of-plane magnetization for clusters with $\bar{n}=4$ and 8 atoms measured at $T=10.0 \pm 0.5 \mathrm{~K}$ (Fig. 2, B and C). The values of $\mathbf{L}$ derived from Eq. 1 are shown in Fig. $3 \mathrm{~A}$ as
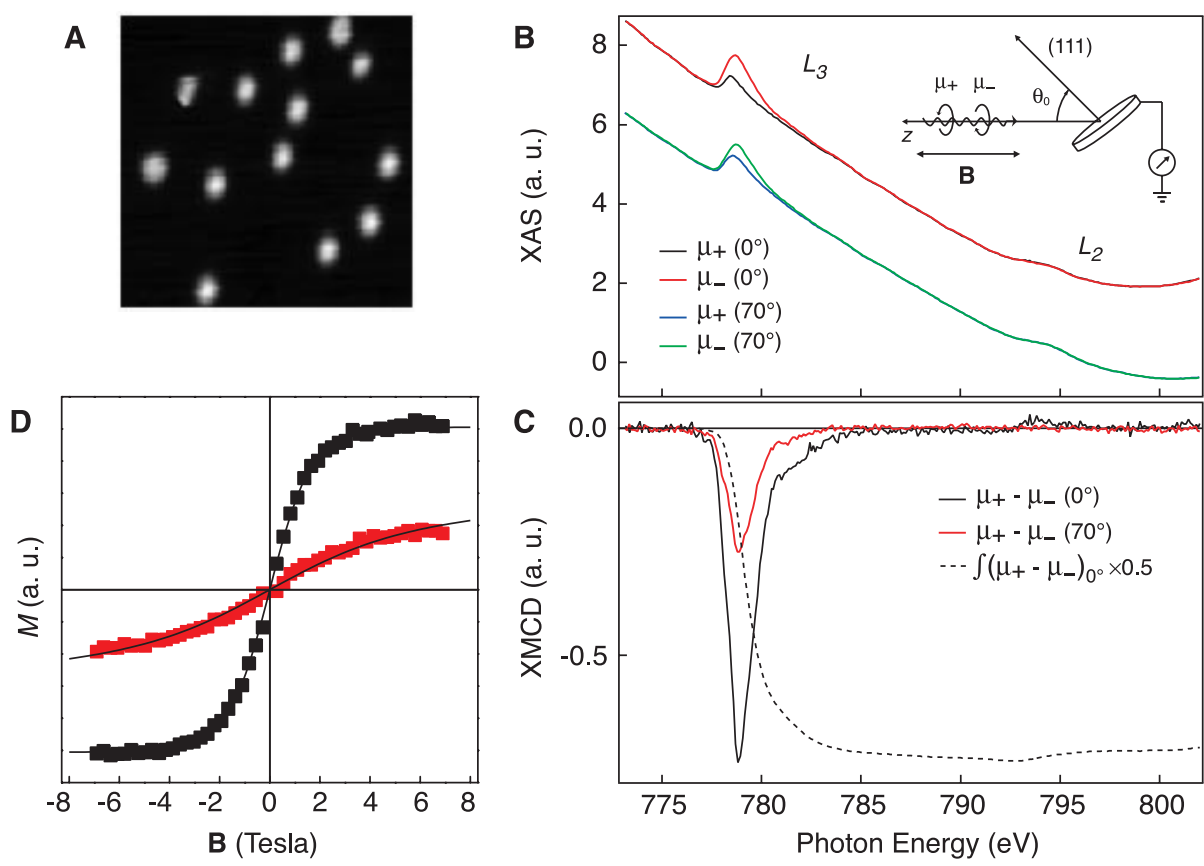

Fig. 1. (A) STM image of isolated Co adatoms (bright dots) on $\mathrm{Pt}(111)$. The Co coverage is 0.010 $\mathrm{ML}$, and the image size is $85 \AA$ by $85 \AA$. (B) $L_{2,3}$ XAS spectra of isolated Co adatoms $(0.010 \mathrm{ML})$ at $T=5.5 \pm 0.5 \mathrm{~K}, \mathbf{B}=7 \mathrm{~T}$ taken with parallel $\left(\mu_{+}\right)$and antiparallel $\left(\mu_{-}\right)$alignment of light helicity with respect to $B$ at $\theta_{0}=0^{\circ}, 70^{\circ}$ relative to the surface normal (inset). The spectra at $70^{\circ}$ have been normalized to the $\left(\mu_{+}+\mu_{-}\right) L_{3}$ intensity at $0^{\circ}$ to eliminate the dependence of the electron yield on the sample orientation. (C) XMCD spectra $\left(\mu_{+}-\mu_{-}\right)$obtained for the $\theta_{0}=0^{\circ}$ and $70^{\circ}$ magnetization directions. The dashed line is the integrated XMCD at $\theta_{0}=0^{\circ}$. (D) Magnetization curves at $\theta_{0}=0^{\circ}$ (black squares) and $70^{\circ}$ (red squares) measured at $T=5.5 \mathrm{~K}$. The points represent the peak of the $L_{3} \mathrm{XMCD}$ intensity at $778.6 \mathrm{eV}$ divided by the pre-edge intensity at $775 \mathrm{eV}$ as a function of B. The difference between the $\theta_{0}=0^{\circ}$ and $70^{\circ}$ curves was checked for consistency with the XAS-normalized XMCD spectra. The solid lines are fits to the data according to Eq. 3. 
a function of $\bar{n}$ (26). The smaller $\bar{n}$ is, the larger the $\mathbf{L}$ dependence on the atomic coordination. Particles with $\bar{n}=3$ and 4 atoms have $\mathbf{L}$ reduced to $0.78 \pm 0.05$ and $0.59 \pm 0.05 \mu_{\mathrm{B}} /$ atom, respectively. The values of $K$ (Fig. 3B) reveal large changes of the MAE for one-atom variations of the particle size. At $\bar{n}=3$ atoms, $K=3.3 \pm 0.4 \mathrm{meV} /$ atom, which is about $30 \%$ of the single-adatom value. The comparison between Fig. 3, A and B, also shows the correlation between $\mathbf{L}$ and $K$, which has been made explicit in the inset of Fig. 3A (filled squares). Treating the spin-orbit coupling in second-order perturbation theory yields a relation $(3,4)$ where $K$ is given by the sum of a term proportional to the anisotropy of the orbital moment $\Delta \mathbf{L}=\mathbf{L}\left(0^{\circ}\right)-\mathbf{L}\left(70^{\circ}\right)$ and a term proportional to $\mathbf{S} \cdot \mathbf{D}$. Because Eq. 1 should be applied at the saturation magnetization to obtain $\mathbf{L}\left(70^{\circ}\right)$, which is not attained for $\mathbf{B}=7 \mathrm{~T}, \Delta \mathbf{L}$ cannot be directly measured. However, the ratio $r=$ $\mathbf{L} /(\mathbf{S}+7 \mathbf{D})$ can be taken as an estimate of $\mathbf{L}$ even away from saturation, provided that $\mathbf{S}$ varies slowly with $\bar{n}$ with respect to $\mathbf{L}$ and $7 \mathbf{D}$ is small compared with $\mathbf{S}$. A plot of $K$ against changes in $r$ as a function of angle ( $\Delta r$; Fig. 3A inset, open diamonds) suggests a linear dependence of the MAE on $\Delta \mathbf{L}$, as expected when $\mathbf{D}$ is small (4), for $\bar{n} \geq 3$.

The experimental results presented above have been compared with ab initio calculations using the spin-polarized-relativistic KorringaKohn-Rostocker (SPR-KKR) Green's function LSDA method (9). In this scheme, the spinpolarized Dirac Green's function is determined by assuming spherical potentials, including the full charge density. The electronic structure of the Co adatoms and Pt substrate atoms has been calculated self-consistently. Lattice relaxations are neglected. The resulting $\mathbf{S}, \mathbf{L}$, and $K$ per Co atom have been obtained for particles with up to five atoms (Fig. 4). We find that $\mathbf{S}$ is very stable and nearly independent of the coordination because the majority spin band is almost filled in all cases. In contrast to $\mathbf{S}, \mathbf{L}$ decreases strongly with increasing Co coordination, from $0.60 \mu_{B}$ for the single Co adatom to $0.22 \mu_{\mathrm{B}} /$ atom for the tetramer. Although this is in qualitative agreement with the experimental results shown in Fig. $3 \mathrm{~A}$, the values are too small by about a factor of 2. Closer agreement with the experiment can be found by calculating $\mathbf{L}$ within the orbital polarization (OP) scheme (27), which is known to improve $\mathbf{L}$ of bulk systems and of the surfaces of elemental ferromagnets. Using a 50\% reduced Racah parameter in the OP term we find, e.g., that $\mathbf{L}$ increases to $1.50,0.74$, and $0.67 \mu_{\mathrm{B}}$ /atom for the monomer, dimer, and trimer chain, respectively. In agreement with the experiment, all Co particles prefer a moment orientation perpendicular to the substrate. The values of $K$ show the correct decrease with cluster size as well as the correct order of magnitude compared with the experimental results. Dimer and trimer chainlike structures also possess large in-plane anisotro-

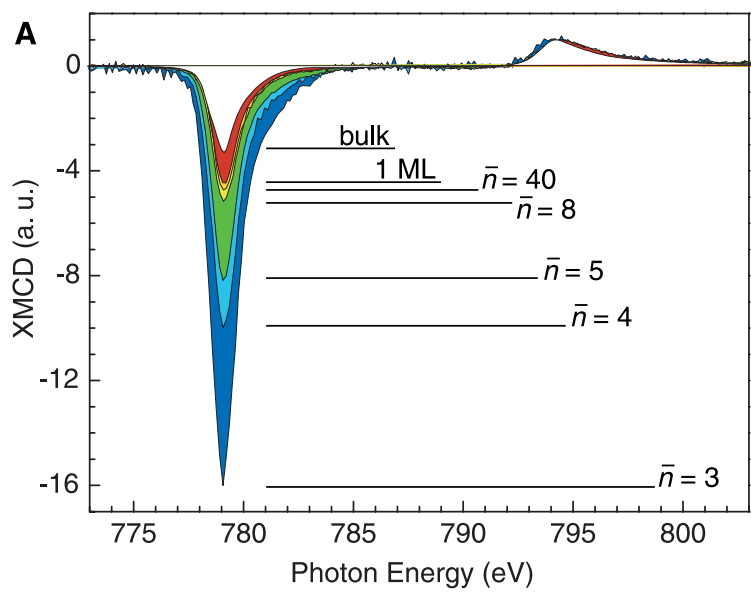

Fig. 2. (A) XMCD spectra as a function of the average particle size $\bar{n}$ (Co coverage 0.10 to 0.20 $\mathrm{ML})$. The spectra have been normalized to the $L_{2}$ intensity to show the reduced weight of the $L_{3}$ component with increasing $\bar{n}$. (B) Magnetization of an assembly of particles with $\bar{n}=4$ atoms at $\theta_{0}=0^{\circ}$ (black squares) and $70^{\circ}$ (red squares) at $T=10 \mathrm{~K}$. The solid lines are fits to the data according to Eq. 3. (C) Same as (B) with $\bar{n}=8$ atoms. Note that the MAE diminishes with respect to (B) and the quality of the fit degrades with respect to that in Fig. 1D as the particle size distribution broadens.
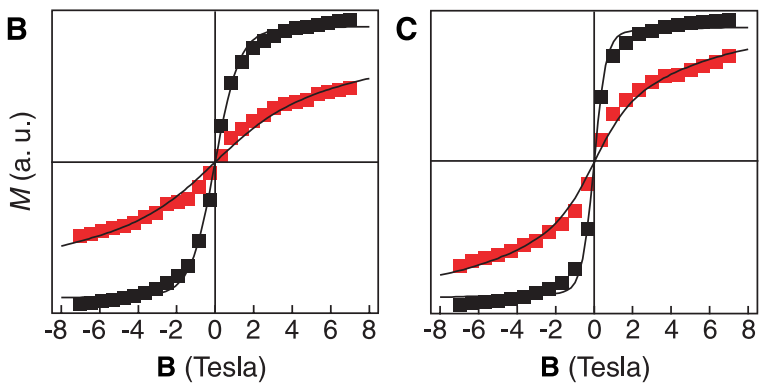

pies with $K_{y-x}=6.91$ and $6.19 \mathrm{meV} /$ atom, respectively, which the present experiment does not allow one to test. By comparing particles with different shape, e.g., the tetramer and the pentamer (Fig. 4B), the calculations show that

the atomic coordination has a stronger influence on $\mathbf{L}$ and $K$ than the absolute particle size. No correlation is found between the particle pointgroup symmetry $\left(\mathrm{C}_{3 v}\right.$ for the monomer and compact trimer, $\mathrm{C}_{2 v}$ for the remaining particles) and

Fig. 3. (A) $\mathbf{L}$ as a function of $\bar{n}$ measured along the easy magnetization direction $\left(\theta_{0}=0^{\circ}\right)$. (B) $\mathrm{K}$ as a function of $\bar{n}$. For comparison, the dashed and dashed-dotted lines show the MAE per Co atom of the $\mathrm{L} 1_{\mathrm{O}}$ CoPt alloy and hcp-Co, respectively. The values of $n$ were determined in situ by fitting the superparamagnetic response of each particle assembly by means of Eq. 3. The average sizes so obtained are within $\pm 10 \%$ of those determined by STM for the same growth conditions. For a given size, the particles consist of different isomers. Particles with $5<\bar{n} \leq 40$ have a compact shape. The Co interatomic distance is that of the underlying Pt lattice. The error bars on the horizontal scale in (A) and (B) represent the standard deviation of the size distribution determined by STM. (Inset) $K$ is plotted as a function of $\mathbf{L}$ (filled squares) and as a function of $\Delta r=r\left(0^{\circ}\right)-r\left(70^{\circ}\right)$ for $\bar{n}>1$ (open diamonds). $\Delta r=$ 0.1 corresponds to about $\Delta \mathrm{L}=$ $0.2 \mu_{B}$; the errors on $\Delta r$ (not shown) are on the order of \pm 0.04 . The lines are guides to the eye.
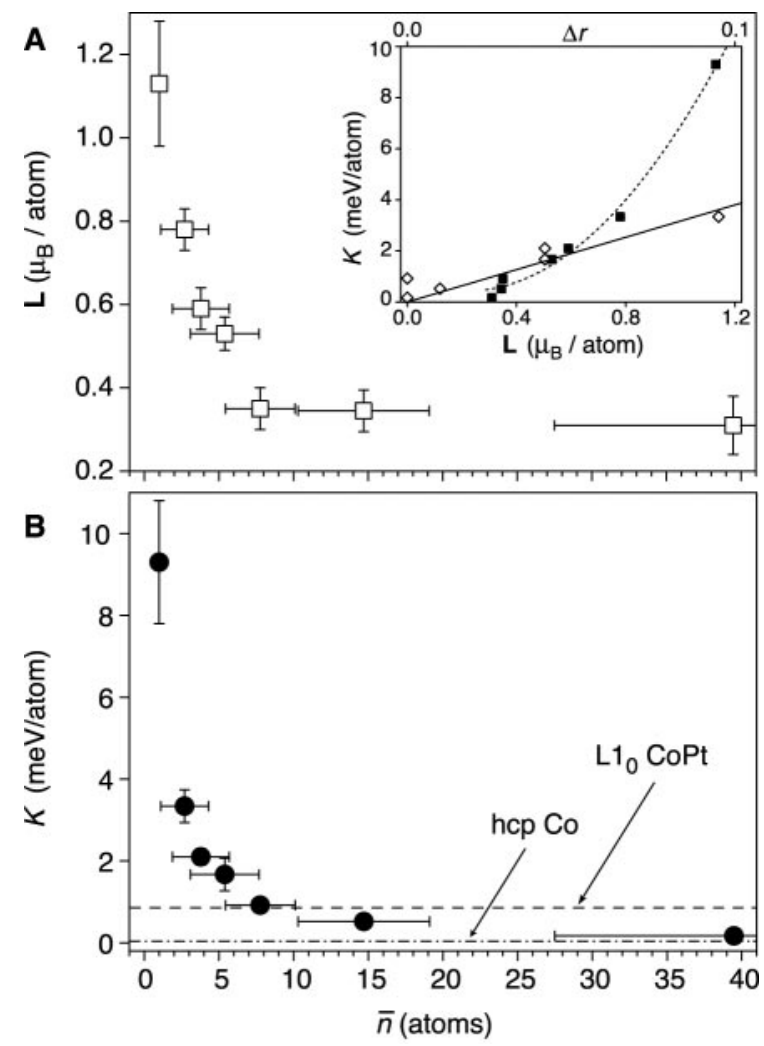
A

\begin{tabular}{ccccc}
\hline$n$ & $\mathbf{S}$ & $\mathbf{L}$ & $\Delta \mathrm{L}_{x-z}$ & $K_{x-z}$ \\
\hline 1 & 2.14 & $0.60(1.50)$ & -0.25 & +18.45 \\
2 & 2.11 & $0.38(0.74)$ & -0.11 & +4.11 \\
3 chain & 2.08 & $0.34(0.67)$ & -0.06 & +3.69 \\
3 triangle & 2.10 & $0.25(0.43)$ & -0.05 & +2.22 \\
4 & 2.08 & $0.22(0.33)$ & -0.01 & +0.75 \\
5 & 2.08 & $0.27(0.45)$ & -0.09 & +1.81 \\
\hline
\end{tabular}

B

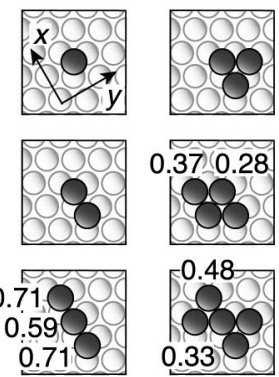

Fig. 4. (A) Values of $\mathbf{S}, \mathbf{L}, \Delta \mathbf{L}\left(\mu_{B}\right)$, and $K(\mathrm{meV})$ per Co atom calculated by the SPR-KKR method for Co particles on $\mathrm{Pt}(111)$ as shown in (B). The values of $L$ in parentheses have been computed within the OP scheme with a 50\% reduced Racah parameter. (B) Hard-sphere representation of the Co particles considered in the theoretical calculations. The labels indicate the OP values of $\mathbf{L}$ for nonequivalent $C o$ sites. $\mathbf{S}, \mathrm{L}, \Delta \mathrm{L}$, and $K$ in $(\mathrm{A})$ are averaged over all Co sites.

changes of $\mathbf{L}$ and $K$. Further, to estimate the MAE due to the induced polarization of the $\mathrm{Pt}$ substrate, $K$ can be decomposed in partial contributions arising from Co and Pt sites (28). For a single Co adatom, Pt sites contribute to about $15 \%$ of the total MAE. Because of the strong decrease of the Co MAE with increased coordination, the $\mathrm{Pt}$ share increases to $30 \%$ for the dimer and up to $60 \%$ for the pentamer, eventually providing the dominant MAE contribution in $\mathrm{Co} / \mathrm{Pt}$ multilayers (25).

These results provide a fundamental understanding of the magnetic properties of finite-sized particles and enable the testing of current theoretical models at the atomic scale $(9,29)$. It is expected that the size of stable ferromagnetic particles at room temperature can be made smaller by artificially reducing the coordination of the magnetic atoms in nanosized particles. If the Co adatom coordination is assumed to equal 2 , the present data imply a theoretical lower limit of $400 \mathrm{Co}$ atoms per bit (30).

References and Notes

1. S. Chikazumi, Physics of Ferromagnetism (Clarendon, Oxford, ed. 2, 1997).

2. D. Weller, A. Moser, IEEE Trans. Mag. 35, 4423 (1999).

3. P. Bruno, Phys. Rev. B 39, 865 (1989).

4. G. van der Laan, J. Phys. Condens. Matter 30, 3239 (1997).

5. S. Sun et al., Science 287, 1989 (2000).

6. H. A. Dürr et al., Phys. Rev. B 59, R701 (1999).

7. A. C. Hewson, The Kondo Problem to Heavy Fermions (Cambridge Univ. Press, Cambridge, 1993).

8. P. Gambardella et al., Phys. Rev. Lett. 88, 047202 (2002).

9. B. Nonas et al., Phys. Rev. Lett. 86, 2146 (2001).

10. I. M. L. Billas, A. Chàtelain, W. A. de Heer, Science 265, 1682 (1994).

11. The room temperature MAE of bulk hcp-Co is $0.45 \times$ $10^{7} \mathrm{erg} / \mathrm{cm}^{3}=0.045 \mathrm{meV} /$ atom $\left(0.70 \times 10^{7} \mathrm{erg} / \mathrm{cm}^{3}\right.$ at $T=4 \mathrm{~K}$ ), compared with, e.g., $0.005 \mathrm{meV} /$ atom for bcc-Fe. The CoPt bulk-ordered $\mathrm{L}_{\mathrm{O}}$ phase has a MAE of $4.9 \times 10^{7} \mathrm{erg} / \mathrm{cm}^{3}=0.8 \mathrm{meV} / \mathrm{Co}$ atom $(1,2)$.

12. H. Röder, E. Hahn, H. Brune, J. P. Bucher, K. Kern, Nature 366, 141 (1993).

13. H. Brune, Surf. Sci. Rep. 31, 121 (1998)

14. J. Stöhr, J. Mag. Mag. Mater. 200, 470 (1999).

15. B. T. Thole, P. Carra, F. Sette, G. van der Laan, Phys. Rev. Lett. 68, 1943 (1992).

16. P. Carra, B. T. Thole, M. Altarelli, X. Wang, Phys. Rev. Lett. 70, 694 (1993)

17. C. T. Chen et al., Phys. Rev. Lett. 75, 152 (1995).

18. T. Koide et al., Phys. Rev. Lett. 87, 257201 (2001).
19. P. Gambardella et al., Nature 416, 301 (2002).

20. R. Wu, A. J. Freeman, Phys. Rev. Lett. 73, 1994 (1994).

21. The magnetization of single atoms is usually described by a Brillouin function. Hybridization of the Co states with the Pt bands, however, produces broad resonances characterized by noninteger $\mathbf{S}$ and $\mathbf{L}$ values, so that a continuum description is more appropriate.

22. N. Nakajima et al., Phys. Rev. Lett. 81, 5229 (1998).
23. D. Weller et al., Phys. Rev. Lett. 75, 3752 (1995).

24. J. A. Mydosh, G. J. Nieuwenhuys, in Ferromagnetic Materials, E. P. Wohlfarth, Ed. (North-Holland, Amsterdam, 1980).

25. I. V. Solovyev, P. H. Dederichs, I. Mertig, Phys. Rev. B 52, 13419 (2002)

26. For simplicity, the $\bar{n}>1$ values are derived from the magnetization fits by assuming a fixed total magnetic moment per Co atom of $3.3 \mu_{B}$. This takes into account the decrease of $\mathbf{S}, \mathbf{L}, \mathbf{m}_{\mathrm{Pt}^{\prime}}$ and the number of neighbors per $\mathrm{Co}$ atom with increasing particle size. The total moment is under- and overestimated by about $10 \%$ for $\bar{n}=3$ and 40 , respectively.

27. M. Brooks, Physica B 130, 6 (1985).

28. Note that the substrate also influences the MAE of Co sites because of Co-Pt hybridization.

29. G. M. Pastor, J. Dorantes-Dàvila, S. Pick, H. Dreysse, Phys. Rev. Lett. 75, 326 (1995)

30. This value is calculated by assuming stable ferromagnetic behavior at $T=350 \mathrm{~K}$ for $\bar{n} K / k_{\mathrm{B}} T=43$, as in (2), with $K=3.3 \mathrm{meV} /$ atom.

31. The XMCD measurements were performed at beamlines ID08 and ID12B of the European Synchrotron Radiation Facility in Grenoble. We thank K. Larsson for technical assistance in preparing the experiment. This work was partly supported by the Swiss Science and Technology Council (Top-Nano-21) and the Swiss National Science Foundation.

28 January 2003; accepted 10 April 2003

\title{
The Impact of the Pull of the Recent on the History of Marine Diversity
}

\section{David Jablonski, ${ }^{1 *}$ Kaustuv Roy, ${ }^{2}$ James W. Valentine, ${ }^{3}$ Rebecca M. Price, ${ }^{1}$ Philip S. Anderson ${ }^{1}$}

\begin{abstract}
Up to $50 \%$ of the increase in marine animal biodiversity through the Cenozoic at the genus level has been attributed to a sampling bias termed "the Pull of the Recent," the extension of stratigraphic ranges of fossil taxa by the relatively complete sampling of the Recent biota. However, 906 of 958 living genera and subgenera of bivalve mollusks having a fossil record occur in the Pliocene or Pleistocene. The Pull of the Recent thus accounts for only $5 \%$ of the Cenozoic increase in bivalve diversity, a major component of the marine record, suggesting that the diversity increase is likely to be a genuine biological pattern.
\end{abstract}

The history of global marine biodiversity is controversial because the observed pattern is difficult to separate definitively from sampling biases. During the Cenozoic, however, the taxonomic richness of the marine fauna increases markedly while sampling proxies, such as measures of marine outcrop area, decline and thus diverge from the biodiversity trend. This discordance is taken by some to indicate that the increase is primarily a true biological signal (1). Others invoke additional biases, particularly the "Pull of the Recent," to explain the Cenozoic

${ }^{1}$ Department of Geophysical Sciences, University of Chicago, 5734 South Ellis Avenue, Chicago, IL 60637, USA. ${ }^{2}$ Section of Ecology, Behavior and Evolution, University of California, San Diego, La Jolla, CA 92903, USA. ${ }^{3}$ Department of Integrative Biology, University of California, Berkeley, CA 94720, USA.

*To whom correspondence should be addressed. Email: d-jablonski@uchicago.edu biodiversity rise (2-6). The Pull of the Recent (7) arises from the more complete sampling of the Recent biota, which tends to extend the stratigraphic ranges of geologically young genera or higher taxa to the present day across intervals where fossils of those taxa are lacking, thereby increasing calculated richness in these intervening intervals. Extinct taxa cannot benefit from such complete sampling, resulting in fewer range extensions and, therefore, artificially low diversity in time intervals lacking extant taxa for any taxonomic database founded on first and last occurrences. Here we test the effects of the Pull of the Recent for a major constituent of post-Paleozoic biodiversity, the marine bivalves.

The most direct way to evaluate this effect is to omit the Recent fauna and analyze biodiversity data exclusively from the youngest fossil occurrence of each taxon $(3,8)$. Sepkoski's $(8)$ 